RICYDE. Revista Internacional de Ciencias del Deporte doi: $10.5232 /$ ricyde

Rev. int. cienc. deporte

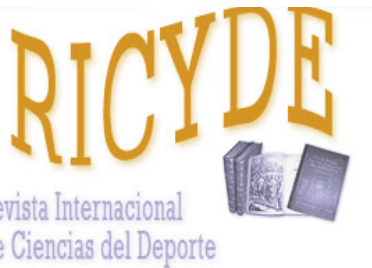

RICYDE. Revista Internacional de Ciencias del Deporte VOLUMEN XII - AÑO XII

Páginas:262-277 ISSN:1885-3137 Número 45 - Julio - 2016

\title{
Clima motivacional de los adolescentes y su relación con el género, la práctica de actividad física, la modalidad deportiva, la práctica deportiva federada y la actividad física familiar
}

Motivational climate of adolescents and their relationship to gender, physical activity, sport, federated sport and physical activity family

\author{
Manuel Castro-Sánchez, Félix Zurita-Ortega, Asunción Martínez-Martínez, \\ Ramón Chacón-Cuberos, Tamara Espejo-Garcés \\ Universidad de Granada. España
}

\begin{abstract}
Resumen
La adolescencia es un periodo crítico en cuanto a la adquisición de patrones de práctica de actividad física que se mantengan durante la etapa adulta, por ello se considera de vital importancia analizar la motivación hacia la asignatura de Educación Física. El presente estudio tiene como objetivo conocer los parámetros de práctica de actividad física y de motivación hacia la asignatura de Educación Física, para analizar las relaciones existentes entre las variables. Participaron 2.134 estudiantes de edades comprendidas entre los 15 y los 18 años, evaluados mediante el cuestionario de Clima Motivacional (PMCQS-2) y una hoja de auto registro que midió las variables físico-deportivas. Los resultados mostraron que tanto los alumnos como las alumnas se orientan más hacia la tarea que hacia el ego, mostrando las chicas valores superiores en el clima tarea, y los chicos en el clima ego. Únicamente un tercio de ellos no practican actividad física, mientras que de los que sí lo hacen, en su mayoría practican deportes colectivos. Dos de cada diez adolescentes se encuentran federados en algún deporte, los varones practican deporte de forma federada en mayor número que las féminas.
\end{abstract}

Palabras clave: clima motivacional; deporte; actividad física; educación física; adolescentes.

\begin{abstract}
Adolescence is a critical regarding the acquisition of patterns of physical activity to be maintained into adulthood, is therefore considered vital to analyze the motivation for Physical Education period. This study aims to determine the parameters of physical activity and motivation towards the subject of Physical Education and analyze the relationships between variables. They involved 2,134 students aged between 15 and 18 years, assessed by questionnaire Motivational Climate (PMCQS-2) and a self-registration sheet which measured the physical and sports variables. The results showed that students are more oriented toward the task to the ego, the girls showing higher values in the job climate, and the guys on the ego climate. Only a third of them do not practice physical activity, while those who if they do, most team sports practice. Two out of ten teenagers are federated in sports, men play a sport on a federated in greater numbers than females.
\end{abstract}

Key words: motivational climate; sport; physical activity; physical education; teenagers. 
Castro-Sánchez, M.; Zurita-Ortega, F.; Martínez-Martínez, A.; Chacón-Cuberos, R., y Espejo-Garcés, T. (2016). Clima motivacional de los adolescentes y su relación con el género, la práctica de actividad física, la modalidad deportiva, la práctica deportiva federada y la actividad física familiar. RICYDE. Revista internacional de ciencias del deporte. 45(12), 262-277. http://dx.doi.org/10.5232/ricyde2016.04504

\section{Introducción}

$\mathrm{L}$ os múltiples beneficios que tiene la realización de actividad física ha aumentado su interés de estudio en numerosos sectores sociales (Estévez-López, Tercedor y DelgadoFernández, 2012; Oviedo y col., 2013). Autores como Gil, Cuevas, Contreras y Díaz (2012) la definen como el movimiento corporal que implica un gasto energético, recomendando la realización de ejercicio moderado varios días semanales. En este sentido, ha tomado fuerza el desarrollo de técnicas de promoción de actividad física en jóvenes, tanto en el contexto educativo como social (Gutiérrez, Ruiz y López, 2011). Según, Zurita y col. (2009), p.285 "hay un consenso por parte de los profesionales de la salud y las autoridades sanitarias de que la actividad física es un factor clave para una buena salud". De hecho, Cantera y Devís-Devís (2002) recuerdan su importancia en la reducción del riesgo de padecer enfermedades y la creación de hábitos de salud. En esta línea, otros de los beneficios que produce un estilo de vida activo son los dados a nivel fisiológico, psicológico o social; abarcando el desarrollo de la capacidad cardiovascular, proporcionando bienestar mental o mejorando las relaciones socio-afectivas (Beltrán-Carrillo, Devís-Devís y Peiró-Velert, 2012; Warburton, Whitney y Bredin, 2006). Estudios como los de Strong y col. (2005) y Hills, King y Amstrong (2007) han proporcionado evidencia científica sobre los beneficios asociados a la práctica de actividad física, como la reducción del riesgo de padecer enfermedades cardiovasculares (British Medical Association Board of Science, 2005), disminución del IMC (Jansen y col., 2005), tratamiento y prevención de la diabetes tipo 2 (Aylin, Williams y Bottle, 2005), además de conllevar beneficios psicosociales como disminución de la ansiedad, de la depresión y de la angustia emocional (Strong y col., 2005). Por ello, Rodríguez, De la Cruz, Feu y Martínez (2012) ponen en relieve la importancia de realizar actividad física en el contexto educativo, ya que los jóvenes pasan en él cerca de un $40 \%$ de su tiempo (Cantera y Devís-Devís, 2002).

La adolescencia constituye una etapa de gran importancia en la creación de hábitos de salud, pues representa el periodo de transición hacia la vida adulta en el que se configuran estilos de vida (González, Garcés y García, 2012) y como propone Gil y col. (2012) es imprescindible indicar el papel de la asignatura de Educación Física en el desarrollo de hábitos físicosaludables, ya que uno de sus ejes esenciales es el de la mejora de las destrezas físicas y motrices, favoreciendo el conocimiento corporal y promoción de la salud (Drake y col., 2012; Sallis y col., 2012). No obstante, la presencia de este área en el programa escolar no asegura un estilo de vida activo, pues los adolescentes se interesan cada vez menos por la misma (Cera, Almagro, Conde y Sáenz-López, 2015; Gutiérrez y col., 2011). En este sentido, los docentes serán fundamentales a la hora de implicar al alumnado en el ejercicio físico a través de la Educación Física (Mora, Cruz y Sousa, 2015), dado que la motivación que proporcionen a los discentes será primordial tanto en la implicación actual como en hábitos futuros (Almagro, Sáenz-López y Moreno-Murcia, 2012).

El papel del docente de educación Física será determinante en la configuración de un clima motivacional óptimo, siendo requisitos indispensables el feedback, las tareas que favorezcan la autonomía, el trabajo en equipo y actividades lúdicas (Cervelló y col., 2011; Conde y Almagro, 2013; Morgan, Kingston y Sproule, 2005).

Estudios sobre la motivación la concretan cómo un elemento sustancial en cualquier proceso del aprendizaje (Mora y col., 2015; Moreno, Jiménez, Gil, Aspano y Torreno, 2011). Según esta perspectiva, un alumnado motivado logrará un aprendizaje más significativo en el contexto educativo, ya que los propios objetivos representarán sus metas a alcanzar (Van Laar, Derks y Ellemers, 2013). De este modo, el clima motivacional creado en el aula posee 
Castro-Sánchez, M.; Zurita-Ortega, F.; Martínez-Martínez, A.; Chacón-Cuberos, R., y Espejo-Garcés, T. (2016). Clima motivacional de los adolescentes y su relación con el género, la práctica de actividad física, la modalidad deportiva, la práctica deportiva federada y la actividad física familiar. RICYDE. Revista internacional de ciencias del deporte. 45(12), 262-277. http://dx.doi.org/10.5232/ricyde2016.04504

una gran importancia en la motivación, pues se define como el ambiente constituido mediante señales explícitas e implícitas en un entorno, las cuales, permiten constituir patrones de éxito (Cera y col., 2015; Méndez-Giménez, Fernández-Río y Cecchini-Estrada, 2014; Moreno y col., 2011).

Según Galván, López-Walle, Pérez, Tristán, y Medina (2013), los elementos que componen el clima motivacional son el uso de recompensas, el diseño de tareas, las agrupaciones y la evaluación realizada por el docente. Por tanto, en función de cómo se utilicen dichos elementos, se podrán generar climas orientados a la maestría o climas orientados al rendimiento (Cuevas, García-Calvo y Contreras, 2013). Son varios los análisis que determinan correspondencia entre la motivación y actividad física (Conde y Almagro, 2013; Owen, Astell-Burt y Lonsdale, 2013; Spittle y Byrne, 2009). Más concretamente, Almagro, Sáenz-López, González-Cutre y Moreno-Murcia (2011) y Carriedo, González y López (2013) estudian el clima motivacional dentro del área de Educación Física, dando consignas generales sobre la concreción de un clima motivacional adecuado basado en la motivación intrínseca (Méndez-Giménez, Cecchini, Fernández-Río y González, 2012). En relación, el Clima Tarea se corresponde con una buena actitud del alumno hacía la asignatura y el deseo de realizar tareas desafiantes, caracterizandose por satisfacer las necesidades de los discentes, la superación personal y la cooperación; reconociendo el esfuerzo sobre los resultados como meta de éxito (Cervelló, Moreno, Martínez, Ferriz y Moya, 2011; Gutiérrez y col., 2011; Moreno y Hellín, 2007), originando actitudes favorables hacia el ejercicio (Galván y col., 2011). Por el contrario, las sesiones orientadas al Clima Ego se relacionan con la consecución de logros sin esfuerzo, fijando poca motivación e interés en las sesiones de Educación Física (Cuevas y col., 2013; Teixeira, Carraça, Markland, Silva y Ryan, 2012), añaden que éste clima suele caracterizarse por la monotonía de las tareas, un estilo autoritario, la comparación social y la búsqueda de reconocimiento externo. El docente de Educación Física es uno de los más influyentes en la etapa adolescente, siendo la asignatura por la que sienten mayor satisfacción (Martín-Albo, 2000; Moreno, 1999), debido a la mayor participación del alumnado en las actividades realizadas en la asignatura, al sentimiento de competencia generado y a la entrega y persistencia en la tarea.

La motivación hacia la actividad física no depende solo del clima motivacional, sino que lo hace de otros componentes al ser un constructo multifactorial (Mora y col., 2015). Algunos de los principales factores motivacionales que pueden determinar, tanto de forma intrínseca como extrínseca, una actitud favorable hacía la práctica de actividad física (Gavita, 2006; Kilpatrick, Hebert y Bartholomew, 2015; Owen y col., 2013; Spittle, y Byrne, 2009), son: la competición, estando en relación con el desarrollo de habilidades; la mejora de la imagen corporal o apariencia; la búsqueda de diversión y situaciones de esparcimiento; la relación social; los beneficios psicológicos, como la liberación de energía y estrés; o la adicción y el exhibicionismo. En este sentido, teniendo en cuenta la importancia de la motivación en la práctica de actividad física, numerosas investigaciones han centrado su interés en la búsqueda de climas motivacionales que generen actitudes positivas hacia el ejercicio (Almagro y col., 2011; Méndez-Giménez y col., 2014; Standage, Gillison, Ntoumanis y Treasure, 2012; Wallhead, Garn y Vidoni, 2014). De acuerdo con lo expresado, Cervelló y col. (2011) relacionaron hace unos años el clima motivacional, las metas de predicción del Flow -o satisfacción-, y la actividad física; destacando la importancia de la predisposición del discente, el clima motivacional creado por el profesor y las relaciones entre pares, con el fin de establecer estados psicológicos adecuados (McDavid, Cox y Amorose, 2011). En relación a esto, se demuestra también que la percepción del clima motivacional varía en función de la 
edad, destacando la importancia de modificar los estímulos del entorno progresivamente (Moreno y Hellín, 2007; Reigal, Videra, Parra, y Juárez, 2012).

Dada la importancia de la práctica de actividad física y su relación con la salud, es imprescindible conocer los patrones de realización de actividad física de los adolescentes, con el fin de paliar su abandono, por ello se plantean los siguientes objetivos para el presente estudio:

- Conocer y determinar los parámetros de práctica de actividad física y de motivación hacia la asignatura de Educación Física.

- Analizar las relaciones entre las variables relacionadas con la práctica de actividad física y la motivación hacia la Educación Física en adolescentes.

\section{Material y Métodos}

\section{Diseño}

En este estudio se utilizó un diseño exploratorio en el que se desarrolló un estudio relacional para analizar el grado de dependencia entre las distintas variables objeto de estudio.

\section{Participantes}

La muestra del estudio estuvo compuesta por un total de 2.134 adolescentes españoles de la provincia de Granada, con edades comprendidas entre los 15 y 18 años ( $M=15$,93 años; $\mathrm{DT}=$ $0,853)$, de los cuales 1.062 eran chicos $(49,8 \%)$ y 1.072 eran chicas $(50,2 \%)$. La selección de la muestra se llevó a cabo mediante un muestreo aleatorio por conglomerados,. Cabe destacar que tanto los centros educativos, como los estudiantes colaboraron voluntariamente en el desarrollo de la investigación.

\section{Variables e instrumentos}

Género, según masculino o femenino.

Práctica de Actividad Física, con la opción dicotómica de si práctica o no, utilizándose una hoja de registro para ello, donde se instaba a marcar la opción de $\mathbf{S i}$, siempre y cuando el adolescente realizase Actividad Física fuera del Centro Escolar con una frecuencia igual o superior a tres horas semanales y No, si la frecuencia era inferior a 3 horas semanales.

Modalidad y tipo de Actividad Física, siguiendo la clasificación propuesta por Álvaro (2015), en la que se plantean cinco opciones de respuesta, empleándose para ello una hoja de autorregistro, donde se planteaban cinco modalidades cada una de ellas con ejemplos para evitar errores de apreciación, de forma que las categorías planteadas eran: a) No Practica, b) Deporte Individual (tenis, atletismo, natación, escalada, ciclismo, ...), c) Deporte Colectivo (futbol, baloncesto, balonmano, voleibol, rugby, waterpolo,....), d) Actividades Gimnasio (artes marciales, spinning, pesas, aerobic,.....) y e) Otros (realizan actividades variadas pero no con regularidad).

Federado, con la opción dicotómica de Si esta federado o No, instando al adolescente a responder a la cuestión en referencia a los dos últimos años.

Nivel de Actividad Física Familiar, dividida en dos opciones, la opción de Si, siempre y cuando uno de los dos progenitores realizase Actividad Física con una frecuencia igual o superior a tres horas semanales y No, si la frecuencia era inferior a 3 horas semanales. 
Clima Motivacional (PMCSQ-2), está extraído de la versión original "Cuestionario de Clima Motivacional Percibido en el Deporte" de Newton, Duda y Yin (2000) y adaptada al español y a la Educación Física por González-Cutre, Sicilia y Moreno (2008), donde mediante una escala Likert de cinco opciones que va desde el $1=$ Totalmente en Desacuerdo hasta el 5= Totalmente de Acuerdo, se valoran 33 ítems. Asimismo este test queda establecido en dos categorías Clima Tarea (Aprendizaje Cooperativo, Esfuerzo/Mejora y Papel Importante) y Clima Ego (Castigo por Errores, Reconocimiento Desigual y Rivalidad entre los Miembros del Grupo). La consistencia interna (alfa de Cronbach) del estudio, obtuvo un $\alpha=.765$ para el cuestionario en general, mientras que para el Clima Tarea se obtuvo un $\alpha=.851$ y en Clima Ego un $\alpha=.867$.

\section{Procedimiento}

Para poder realizar correctamente la recogida de datos, se contactó con la Delegación de Educación y se solicitó la colaboración de los Centros Educativos. Se informó de la naturaleza de la investigación a los responsables de cada uno de los centros educativos, pidiendo su colaboración y la de sus alumnos, y se les entregó un modelo de autorización dirigida a sus tutores legales pidiendo el consentimiento informado de la participación en el estudio. Los investigadores estuvieron presentes durante el proceso de recogida de los datos, con una duración de 20 minutos por grupo de alumnos, informando sobre la manera correcta de cumplimentar los cuestionarios, garantizando el anonimato de los datos recogidos en la investigación.

El estudio respeta el resguardo a la confidencialidad y cumple con las normas éticas del Comité de Investigación y Declaración de Helsinki de 1975.

Los análisis estadísticos del presente estudio se realizaron utilizando el paquete estadístico SPSS 22.0. En primer lugar, se calcularon los estadísticos descriptivos de las diferentes variables de estudio mediante la utilización de medias y frecuencias. A continuación se realizó el estudio relacional empleando tablas de contingencia y ANOVA.

\section{Resultados}

Tomando como referencia los datos de la tabla 1, la muestra presentaba proporciones similares por género, siendo el 49,8\% $(n=1062)$ varones y el 50,2\% $(n=1072)$ féminas. El $32,9 \%$ de los adolescentes no practicaban actividad física, el 27,2\% $(\mathrm{n}=581)$ practicaban deportes colectivos, el $14,4 \%(n=307)$ deportes individuales y únicamente un $4,3 \%(n=91)$ realizaban actividades deportivas de gimnasio relacionadas con el fitness. De los adolescentes encuestados, solamente el $21,9 \%(n=452)$ estaban federados en algún deporte. En cuanto al clima motivacional, el Clima Ego obtuvo valores medios de 2,63, valorándose de forma inferior al Clima Tarea, con una media de 3,56. Las categorías del Clima Ego, Castigo por Errores, Reconocimiento Desigual y Rivalidad entre Miembros del Grupo, obtuvieron valores similares a los obtenidos en el Clima Ego. Y los valores obtenidos en las categorías del Clima Tarea, Aprendizaje Cooperativo, Esfuerzo/Mejora, y Papel Importante fueron casi idénticos a los obtenidos en el Clima Tarea. 
Castro-Sánchez, M.; Zurita-Ortega, F.; Martínez-Martínez, A.; Chacón-Cuberos, R., y Espejo-Garcés, T. (2016). Clima motivacional de los adolescentes y su relación con el género, la práctica de actividad física, la modalidad deportiva, la práctica deportiva federada y la actividad física familiar. RICYDE. Revista internacional de ciencias del deporte. 45(12), 262-277. http://dx.doi.org/10.5232/ricyde2016.04504

Tabla 1. Descriptivos de las variables.

\begin{tabular}{|c|c|c|c|}
\hline & Masculino & \multicolumn{2}{|c|}{$49,8 \%(n=1062)$} \\
\hline & Femenino & \multicolumn{2}{|c|}{$50,2 \%(\mathrm{n}=1072)$} \\
\hline \multirow{5}{*}{$\begin{array}{c}\text { Modalidad } \\
\text { Deportiva }\end{array}$} & Deportes Individuales & \multicolumn{2}{|c|}{$14,4 \%(n=307)$} \\
\hline & Deportes Colectivos & \multicolumn{2}{|c|}{$27,2 \%(\mathrm{n}=581)$} \\
\hline & Actividades de Gimnasio & \multicolumn{2}{|c|}{$4,3 \%(n=91)$} \\
\hline & Otros & \multicolumn{2}{|c|}{$21,2 \%(n=452)$} \\
\hline & No practica & \multicolumn{2}{|c|}{$32,9 \%(n=703)$} \\
\hline \multirow{2}{*}{ Federado } & $\mathrm{Si}$ & \multicolumn{2}{|c|}{$21,9 \%(n=467)$} \\
\hline & No & \multicolumn{2}{|c|}{$78,1 \%(n=1667)$} \\
\hline \multirow{3}{*}{ A.F. Padres } & $\mathrm{Si}$ & \multicolumn{2}{|c|}{$51,8 \%(\mathrm{n}=1105)$} \\
\hline & No & \multicolumn{2}{|c|}{$48,2 \%(n=1029)$} \\
\hline & & Media & D. $\mathbf{T}$. \\
\hline \multirow{8}{*}{$\underset{\text { Motivacional }}{\text { Clima }}$} & Clima Ego & 2.63 & .7299 \\
\hline & Castigo por errores & 2.55 & .8437 \\
\hline & Reconocimiento desigual & 2.65 & .9019 \\
\hline & Rivalidad entre miembros grupo & 2.68 & .8881 \\
\hline & Clima Tarea & 3.56 & .6626 \\
\hline & Aprendizaje cooperativo & 3.52 & .8370 \\
\hline & Esfuerzo/Mejora & 3.65 & .7085 \\
\hline & Papel importante & 3.51 & .7962 \\
\hline
\end{tabular}

Atendiendo a la tabla 2, no se ha encontrado asociación estadística al relacionar el género con la modalidad deportiva practicada por los adolescentes $(p=.917)$, ni al relacionarlo con la práctica de actividad física de los padres $(p=.801)$. Sin embargo, al relacionar el género con estar federado en algún deporte sí se han encontrado diferencias estadísticamente significativas $(p=.003)$, éstas asociaciones vienen dadas porque hay más varones federados que féminas, encontrando que el 24,6\% $(n=261)$ de los jóvenes, se encuentran federados, frente a las chicas, en las que éstas cifras disminuyen, obteniéndose en ellas un 19,2\% $(n=206)$. 
Castro-Sánchez, M.; Zurita-Ortega, F.; Martínez-Martínez, A.; Chacón-Cuberos, R., y Espejo-Garcés, T. (2016). Clima motivacional de los adolescentes y su relación con el género, la práctica de actividad física, la modalidad deportiva, la práctica deportiva federada y la actividad física familiar. RICYDE. Revista internacional de ciencias del deporte. 45(12), 262-277. http://dx.doi.org/10.5232/ricyde2016.04504

Tabla 2. Modalidad deportiva, Federado y Actividad física de los padres según género.

\begin{tabular}{|c|c|c|c|c|}
\hline & & \multicolumn{2}{|c|}{ Género } & \multirow[b]{2}{*}{$p$} \\
\hline & & Masculino & Femenino & \\
\hline \multirow{5}{*}{$\begin{array}{c}\text { Modalidad } \\
\text { Deportiva }\end{array}$} & Deportes Individuales & $14,7 \%(\mathrm{n}=156)$ & $14,1 \%(n=151)$ & \multirow{5}{*}{.917} \\
\hline & Deportes Colectivos & $27,2 \%(n=289)$ & $27,2 \%(n=292)$ & \\
\hline & Actividades Gimnasio & $3,9 \%(n=41)$ & $4,7 \%(n=50)$ & \\
\hline & Otros & $21,2 \%(n=225)$ & $21,2 \%(n=227)$ & \\
\hline & No practica & $33,1 \%(n=351)$ & $32,8 \%(n=352)$ & \\
\hline \multirow{2}{*}{ Federado } & $\mathrm{Si}$ & $24,6 \%(n=261)$ & $19,2 \%(n=206)$ & \multirow{2}{*}{$.003^{*}$} \\
\hline & No & $75,4 \%(n=801)$ & $80,8 \%(n=866)$ & \\
\hline \multirow{2}{*}{ A.F. Familiar } & $\mathrm{Si}$ & $51,5 \%(n=547)$ & $52,1 \%(\mathrm{n}=558)$ & \multirow{2}{*}{.801} \\
\hline & No & $48,5 \%(n=515)$ & $47,9 \%(n=514)$ & \\
\hline
\end{tabular}

Al relacionar el clima motivacional con el género de los adolescentes se ha encontrado asociación estadística en todas las categorías $(p \leq .05)$, excepto en la categoría del Clima Tarea: Papel Importante $(p=.070)$, como se puede comprobar en la tabla 3. En la categoría Clima Tarea y sus categorías: Aprendizaje Cooperativo y Esfuerzo/Mejora, las chicas obtienen mayores puntuaciones que los chicos. Situación que se invierte en el caso del Clima Ego y sus respectivas categorías: Castigo por Errores, Reconocimiento Desigual y Rivalidad entre los Miembros del Grupo, en las que los varones obtienen cifras superiores a las féminas.

Tabla 3. Clima motivacional según género.

\begin{tabular}{|c|c|c|c|c|c|c|}
\hline & \multicolumn{4}{|c|}{ Género } & \multirow{3}{*}{$\mathbf{F}$} & \multirow{3}{*}{$p$} \\
\hline & \multicolumn{2}{|c|}{ Masculino } & \multicolumn{2}{|c|}{ Femenino } & & \\
\hline & Media & D. $\mathbf{T}$. & Media & D. $\mathrm{T}$. & & \\
\hline Clima Ego & 2.70 & .7057 & 2.56 & .7468 & 19.976 & $.000^{*}$ \\
\hline Castigo por errores & 2.60 & .8306 & 2.54 & .8539 & 7.829 & $.005^{*}$ \\
\hline Reconocimiento desigual & 2.71 & .8702 & 2.59 & .9290 & 9.234 & $.002 *$ \\
\hline Rivalidad entre miembros grupo & 2.78 & .8880 & 2.58 & .8772 & 27.768 & $.000^{*}$ \\
\hline Clima Tarea & 3.52 & .6553 & 3.59 & .6681 & 6.193 & $.013 *$ \\
\hline Aprendizaje cooperativo & 3.48 & .8333 & 3.56 & .8392 & 4.925 & $.027 *$ \\
\hline Esfuerzo/Mejora & 3.61 & .6965 & 3.68 & .7187 & 5.380 & $.020^{*}$ \\
\hline Papel importante & 3.48 & .7753 & 3.54 & .8155 & 3.289 & .070 \\
\hline
\end{tabular}

Cuando se relaciona el clima motivacional con la modalidad deportiva en la tabla 4 , no se encuentra asociación estadística en ninguna de las categorías analizadas $(p \geq .05)$. Los adolescentes presentaban cifras muy similares en clima motivacional y sus categorías, independientemente de no realizar actividad física o practicar algún tipo de actividad deportiva. 
Castro-Sánchez, M.; Zurita-Ortega, F.; Martínez-Martínez, A.; Chacón-Cuberos, R., y Espejo-Garcés, T. (2016). Clima motivacional de los adolescentes y su relación con el género, la práctica de actividad física, la modalidad deportiva, la práctica deportiva federada y la actividad física familiar. RICYDE. Revista internacional de ciencias del deporte. 45(12), 262-277. http://dx.doi.org/10.5232/ricyde2016.04504

Tabla 4. Clima motivacional según modalidad deportiva.

\begin{tabular}{|c|c|c|c|c|c|c|c|c|c|c|c|c|}
\hline & \multicolumn{2}{|c|}{$\begin{array}{c}\text { Deportes } \\
\text { Individuales }\end{array}$} & \multicolumn{2}{|c|}{$\begin{array}{c}\text { Deportes } \\
\text { Colectivos }\end{array}$} & \multicolumn{2}{|c|}{$\begin{array}{c}\text { Actividades } \\
\text { Gimnasio }\end{array}$} & \multicolumn{2}{|c|}{ Otros } & \multicolumn{2}{|c|}{ No practica } & \multirow[t]{2}{*}{$\mathbf{F}$} & \multirow{2}{*}{$p$} \\
\hline & Media & D. T. & Media & D. T. & Media & D. T. & Media & D. T. & Media & D. T. & & \\
\hline Clima Ego & 2.63 & .729 & 2.59 & .725 & 2.61 & .805 & 2.64 & .718 & 2.64 & .731 & .464 & .762 \\
\hline $\begin{array}{l}\text { Castigo por } \\
\text { errores }\end{array}$ & 2.55 & .805 & 2.52 & .849 & 2.56 & .872 & 2.54 & .832 & 2.59 & .859 & .546 & .702 \\
\hline $\begin{array}{c}\text { Reconocimiento } \\
\text { desigual }\end{array}$ & 2.67 & .958 & 2.61 & .896 & 2.55 & .959 & 2.67 & .876 & 2.66 & .890 & .667 & .615 \\
\hline $\begin{array}{c}\text { Rivalidad } \\
\text { miembros grupo }\end{array}$ & 2.68 & .879 & 2.65 & .876 & 2.72 & .898 & 2.70 & .886 & 2.69 & .902 & .327 & .860 \\
\hline Clima Tarea & 3.51 & .036 & 3.59 & .026 & 3.59 & .062 & 3.54 & .032 & 3.57 & .025 & .841 & .499 \\
\hline $\begin{array}{l}\text { Aprendizaje } \\
\text { cooperativo }\end{array}$ & 3.50 & .803 & 3.55 & .835 & 3.53 & .737 & 3.49 & .851 & 3.53 & .856 & .425 & .791 \\
\hline Esfuerzo/Mejora & 3.61 & .715 & 3.67 & .681 & 3.67 & .668 & 3.65 & .727 & 3.64 & .721 & .508 & .730 \\
\hline $\begin{array}{c}\text { Papel } \\
\text { importante }\end{array}$ & 3.42 & .756 & 3.54 & .784 & 3.56 & .735 & 3.49 & .815 & 3.53 & .816 & 1.466 & .210 \\
\hline
\end{tabular}

$* p<.05$

En la tabla 5 no se ha encontrado asociación estadística entre estar federado en algún deporte y los niveles de clima motivacional de los adolescentes $(p \geq .05)$. Tampoco se han encontrado diferencias significativas entre la práctica de actividad física de los padres y el clima motivacional de los alumnos $(p \geq .05)$.

Tabla 5. Clima motivacional según práctica de actividad física de los padres y si están federados.

\begin{tabular}{|c|c|c|c|c|c|c|c|c|c|c|c|c|}
\hline & \multicolumn{5}{|c|}{ Federado } & \multicolumn{6}{|c|}{ A.F. Padres } & \multirow{3}{*}{$p$} \\
\hline & \multicolumn{2}{|c|}{$\mathbf{S i}$} & \multicolumn{2}{|c|}{ No } & \multirow{2}{*}{$\mathbf{F}$} & \multirow[t]{2}{*}{$p$} & \multicolumn{2}{|c|}{$\mathbf{S i}$} & \multicolumn{2}{|c|}{ No } & \multirow{2}{*}{$\mathbf{F}$} & \\
\hline & Media & D. T. & Media & D. T. & & & Media & D. T. & Media & D. T. & & \\
\hline Clima Ego & 2.64 & .761 & 2.62 & .720 & .315 & .575 & 2.61 & .723 & 2.65 & .736 & 1.75 & .186 \\
\hline $\begin{array}{l}\text { Castigo por } \\
\text { errores }\end{array}$ & 2.55 & .835 & 2.55 & .846 & .007 & .931 & 2.52 & .851 & 2.58 & .834 & 2.68 & .106 \\
\hline $\begin{array}{c}\text { Reconocimient } \\
\text { o desigual }\end{array}$ & 2.67 & .929 & 2.64 & .894 & .599 & .439 & 2.61 & .889 & 2.68 & .914 & 3.35 & .067 \\
\hline $\begin{array}{c}\text { Rivalidad } \\
\text { miembros } \\
\text { grupo }\end{array}$ & 2.70 & .899 & 2.67 & .885 & .463 & .497 & 2.68 & .890 & 2.68 & .886 & .017 & .897 \\
\hline Clima Tarea & 3.56 & .646 & 3.56 & .667 & .042 & .837 & 3.58 & .648 & 3.54 & .677 & 1.75 & .185 \\
\hline $\begin{array}{l}\text { Aprendizaje } \\
\text { cooperativo }\end{array}$ & 3.52 & .831 & 3.52 & .838 & .001 & .975 & 3.55 & .824 & 3.49 & .849 & 2.78 & .095 \\
\hline $\begin{array}{c}\text { Esfuerzo/Mejo } \\
\text { ra }\end{array}$ & 3.66 & .701 & 3.65 & .710 & .107 & .744 & 3.66 & .707 & 3.63 & .709 & 1.03 & .310 \\
\hline $\begin{array}{c}\text { Papel } \\
\text { importante }\end{array}$ & 3.51 & .766 & 3.51 & .804 & .036 & .850 & 3.52 & .774 & 3.50 & .819 & .426 & .514 \\
\hline
\end{tabular}


Castro-Sánchez, M.; Zurita-Ortega, F.; Martínez-Martínez, A.; Chacón-Cuberos, R., y Espejo-Garcés, T. (2016). Clima motivacional de los adolescentes y su relación con el género, la práctica de actividad física, la modalidad deportiva, la práctica deportiva federada y la actividad física familiar. RICYDE. Revista internacional de ciencias del deporte. 45(12), 262-277. http://dx.doi.org/10.5232/ricyde2016.04504

\section{Discusión}

En la presente investigación, realizada sobre una muestra de 2.134 adolescentes, se ha encontrado que dos tercios de ellos practican actividad física extraescolar de forma regular, aunque únicamente dos de cada diez adolescentes lo hacía de forma federada, siendo los chicos los que practican más deporte federado (Isorna, Rial y Vaquero-Cristóbal, 2014). Se han encontrado resultados similares en cuanto a la práctica de actividad física en los estudios de Ruiz, García y Hernández (2001) y Abarca-Sos, Zaragoza, Generelo y Julián (2010), comprobándose que más del $70 \%$ de la muestra cumple las recomendaciones de práctica de actividad física propuestas por la Organización Mundial de la Salud. Por otro lado encontramos estudios como el de González y Portolés (2014), en el que las cifras de práctica de actividad física son inferiores. Los altos porcentajes de práctica de actividad física encontrados en los adolescentes de la muestra se pueden atribuir a diversas razones: en primer lugar, los ayuntamientos, junto con las concejalías de deporte han aumentado su oferta deportiva en los últimos 10 años, facilitando el acceso la práctica de actividad física y ejercicio con la construcción de nuevas instalaciones como piscinas y pabellones cubiertos, pudiendo refugiarse del frío y días lluviosos; en segundo lugar a que gran parte proviene de zonas costeras con un clima suave durante todo el año, lo que resulta propicio para la práctica de actividad física o deportiva; en tercer lugar, los altos porcentajes de práctica deportiva se deben a la edad de la muestra, siendo la adolescencia la etapa en la que más actividad física o deportiva se practica.

Respecto a las modalidades más practicadas, una cuarta parte de los adolescentes indicaban practicar deportes colectivos, mientras que las actividades de gimnasio son las menos demandadas. Estos resultados concuerdan con los encontrados en la investigación de Luengo (2007), que señalaba que los deportes más practicados son balonmano y fútbol. La adhesión a los deportes colectivos viene motivada por el gran poder mediático del fútbol y el baloncesto, siendo éstos los deportes más practicados a nivel nacional y mundial (López-Alameda y col., 2012; Pedrosa, García-Cueto, Suárez-Álvarez y Pérez, 2012).

En lo concerniente a la práctica de actividad física por los padres, algo más de la mitad de la muestra confesó que sus progenitores practicaban ejercicio de forma habitual, datos que concuerdan con los encontrados en el estudio de Revuelta y Esnaola (2011) en adolescentes procedentes del País Vasco y Cantabria, que nos indican que en torno al 20\% de los padres de adolescentes practican actividad física. Sin embargo, difieren de los datos encontrados por Luengo (2007), que descubrió que los progenitores de un grupo de alumnos de Madrid apenas practicaban deporte. Es muy importante la influencia de la familia para que los adolescentes practiquen deporte y, en general, adquieran hábitos saludables (Pérez y Delgado, 2013). Aquellos adolescentes cuyos padres realizan actividad física, tienen más posibilidad de seguir las pautas que llevan a cabo sus progenitores (Salazar, Vizuete y de la Cruz-Sánchez, 2013). También Fredricks y Eccles (2004), hablan de la importancia del contacto de la persona con el deporte desde edades tempranas, lo cual transmite cantidad de valores de forma directa e indirecta.

En el presente estudio, únicamente el 21,9\% $(\mathrm{n}=452)$ estaban federados en algún deporte, siendo la mayoría de ellos chicos. Los datos obtenidos difieren de los del estudio de Isorna, Rial y Vaquero-Cristóbal (2014), en el que el 52\% de la muestra se encontraban federados en algún deporte, o el estudio de García, Pérez, Rodríguez, Moral y Agudo (2013), en el que únicamente uno de cada diez adolescentes se encontraban federados en algún deporte. En los estudios consultados a nivel nacional se encuentran cifras muy bajas de adolescentes 
Castro-Sánchez, M.; Zurita-Ortega, F.; Martínez-Martínez, A.; Chacón-Cuberos, R., y Espejo-Garcés, T. (2016). Clima motivacional de los adolescentes y su relación con el género, la práctica de actividad física, la modalidad deportiva, la práctica deportiva federada y la actividad física familiar. RICYDE. Revista internacional de ciencias del deporte. 45(12), 262-277. http://dx.doi.org/10.5232/ricyde2016.04504

federados porque en la mayoría de los casos de desconocen los beneficios de federarse en algún deporte o incluso la posibilidad de hacerlo.

Los adolescentes analizados en el presente estudio obtienen puntuaciones más altas en el Clima Tarea y sus tres subcategorías, que en el Clima Ego y las respectivas categorías, indicando que los alumnos dan más importancia al proceso de aprendizaje que al resultado obtenido, basándose en el esfuerzo y la mejora personal (Torregrosa, Sousa, Viladrich, Villamarín y Cruz, 2008). Estos datos coinciden con la totalidad de investigaciones realizadas en contextos similares, como las de González-Cutre, Sicilia y Moreno (2011), Almagro, Sáenz-López y Moreno-Murcia (2012), Martínez, Cervelló y Moreno (2013), MéndezGiménez, Fernández-Rio y Cecchini-Estrada (2013), Moreno-Murcia, Zomeño, Marín, Ruíz y Cervelló (2013) o Moreno-Murcia y col. (2014). Éstas características que favorecen la orientación hacia la tarea, favorecen la implicación de los alumnos en las clases de Educación Física, el esfuerzo, la persistencia y la adherencia a la práctica de actividad física (Moreno, Cervelló y González-Cutre, 2007).

En cuanto al género, la mayoría de investigaciones que han estudiado los niveles de Clima motivacional en relación con el género de los adolescentes, han encontrado que el género femenino obtiene cifras superiores al masculino en la categoría Clima Tarea, situación que se invierte en el Clima Ego, donde ellos obtienen valores más elevados que ellas (Carriedo y col., 2013; Coterón-López, Franco, Pérez-Tejero y Sampedro, 2013; Cuevas y col., 2013; Flórez, Salguero, Molinero y Marquez, 2011; Torregrosa y col., 2011); datos que coinciden con los hallados en la presente investigación. Éstas diferencias que atiende al género de los sujetos han sido explicadas por diversos autores, los cuales atienden a factores sociales que influyen sobre las formas de socialización deportiva en varones y féminas, sospechándose que las féminas interpretan el deporte como una actividad cooperativa orientada al ocio y la recreación, frente a los varones, en los que predomina el factor competitivo asociado al deporte (Moreno-Murcia, Cervelló-Gimeno y González-Cutre, 2008; Vazou, Ntoumanis y Duda, 2006).

En la presente investigación no se ha encontrado relación entre el clima motivacional y la modalidad deportiva practicada. La mayoría de estudios sobre el clima motivacional se realizan en determinados deportes, como el de Zafra (2015), en el que se estudia el clima motivacional mediante un modelo de ecuaciones estructurales en judocas, o el estudio de Balaguer, Castillo, Ródenas, Fabra y Duda (2015), en el que estudian la motivación en futbolistas.

\section{Conclusión}

Como principales conclusiones de este estudio se puede señalar que los adolescentes de Educación Secundaria de la provincia de Granada se orientan más al clima tarea que al clima ego, centrándose en el proceso de aprendizaje más que en el rendimiento. Únicamente un tercio de ellos no practican actividad física, mientras que de los que si lo hacen, en su mayoría practican deportes colectivos, seguido de individuales, encontrando una minoría de ellos que realizan actividades de gimnasio. Dos de cada diez adolescentes se encuentran federados en algún deporte, federándose los varones más que las féminas. La mitad de los padres de estos adolescentes realizan actividad física de forma regular. Asimismo se ha comprobado que no existe relación entre el clima motivacional y la práctica deportiva, ni con la modalidad deportiva practicada. Asimismo tampoco existía asociación entre la motivación y estar federado en algún deporte y la práctica de actividad física de los padres de los adolescentes. 
La presente investigación complementa a numerosos estudios realizados en poblaciones adolescentes sobre el clima motivacional, aportando nuevos datos en una población de Segundo Ciclo de Educación Secundaria Obligatoria, coincidiendo con una de las etapas clave en el desarrollo del adolescente; asimismo entendemos que se deben interpretar los resultados con cierta cautela por tratarse de un estudio de carácter transversal, el cuál no permite establecer relaciones causales. Existe como posibilidad de ampliar la presente investigación, la inclusión del análisis del rendimiento académico de los adolescentes en general y específicamente en la asignatura de Educación Física, lo que aportaría la posibilidad de cotejar dichos datos.

Los hallazgos obtenidos ponen de manifiesto la necesidad de fomentar el Clima Tarea en las clases de Educación Física, con el fin de conseguir una motivación más autodeterminada, y así aumentar la práctica deportiva de los adolescentes y el mantenimiento de ésta durante la adultez, adquiriendo hábitos saludables que mejoren la salud de la población; además de invitar a continuar las investigaciones sobre el clima motivacional en la etapa adolescente.

\section{Referencias}

Abarca-Sos, A.; Zaragoza, J.; Generelo, E., y Julián, J. A. (2010). Comportamientos sedentarios y patrones de actividad física en adolescentes. Revista Internacional de Medicina y Ciencias de la Actividad Física y el Deporte, 10(39), 410-427.

Almagro, B. J.; Sáenz-López, P.; González-Cutre, D., y Moreno-Murcia, J. A. (2011). Clima motivacional percibido, necesidades psicológicas y motivación intrínseca como predictores del compromiso deportivo en adolescentes. RICYDE. Revista Internacional de Ciencias del Deporte, 25(7), 251-265. http://dx.doi.org/10.5232/ricyde2011.02501

Almagro, B.; Sáenz-López, P., y Moreno-Murcia, J. A. (2012). Perfiles motivacionales de deportistas adolescentes españoles. Revista de Psicología del Deporte, 21(2), 223231.

Álvaro, J. (2015). Análisis del autoconcepto en relación con factores educativos, familiares, físico y psicosociales en adolescentes de la provincia de Granada. Tesis Doctoral: Universidad de Granada.

Aylin, P.; Williams, S., \& Bottle, A., (2005). Obesity and type 2 diabetes in children, 1996-7 to 2003-4. British Medical Journal, 331, 1167-1178. http://dx.doi.org/10.1136/bmj.331.7526.1167

Beltrán-Carrillo, V. J.; Devís-Devís, J., y Peiró-Velert, C. (2012). Actividad física y sedentarismo en adolescentes de la comunidad valenciana. Revista Internacional de Medicina y Ciencias de la Actividad Física y Deporte, 12(45), 122-137.

British Medical Association Board of Science. (2005). Preventing Childhood Obesity. London: British Medical Association.

Cantera, M. A., y Devís-Devís, J. (2002). La promoción de la actividad física relacionada con la salud en el ámbito escolar. Implicaciones y propuestas a partir de un estudio realizado entre adolescentes. Apunts. Educación física y deportes, (67), 54-62.

Carriedo, A.; González, C., y López, I. (2013). Relación entre las metas de logro en las clases de educación física y el autoconcepto de los adolescentes. Revista Española de Educación Física y Deportes, 403, 13-24.

Cera, E.; Almagro, B. J.; Conde, C., y Sáenz-López, P. (2015). Inteligencia emocional y motivación en educación física en secundaria. Retos: Nuevas tendencias en Educación Física, Deporte y Recreación, 27, 8-13. 
Cervelló, E. M.; Moreno, J. A.; Martínez, C.; Ferriz, R., y Moya, M. (2011). El papel del clima motivacional, la relación con los demás y la orientación de metas en la predicción del Flow disposicional en educación física. Revista de Psicología del Deporte, 20(1), 165-178.

Conde, C., y Almagro, B. J. (2013). Estrategias para desarrollar la inteligencia emocional y la motivación en el alumnado de educación física. Revista de Educación, Motricidad e Investigación, 1, 212-220.

Coterón-López, J.; Franco, E.; Pérez-Tejero, J., y Sampedro, J. (2013). Clima motivacional, competencia percibida, compromiso y ansiedad en Educación Física. Diferencias en función de la obligatoriedad de la enseñanza. Revista de Psicología del Deporte, 22(1), 151-157.

Cuevas, R.; García-Calvo, T., y Contreras, O. (2013). Perfiles motivacionales en Educación Física: una aproximación desde la teoría de las Metas de Logro 2x2. Anales de Psicología, 29(3), 685-692.

Drake, K. M.; Beach, M. L.; Logacre, M. R.; MacKenzie, T.; Titus, L. J.; Rundle, A. G., \& Dalton, M. A. (2012). Influence of sports, physical education and active commuting to school on adolescent weight status. Pediatrics, 130(2), 296-304. http://dx.doi.org/10.1542/peds.2011-2898

Estévez-López, F.; Tercedor, P., y Delgado-Fernández, M. (2012). Recomendaciones de actividad física para adultos sanos. Revisión y situación actual. Journal of Sport and Health Research, 4(3), 233-244.

Flórez, J. A.; Salguero, A.; Molinero, O., y Márquez, S. (2011). Relación de la habilidad física percibida y el clima motivacional percibido en estudiantes de secundaria, colombianos. Psychologia. Avances de la Disciplina, 5(2), 69-79.

Fredricks, J. A., \& Eccles, J. S. (2004). Parental Influences on Youth Involvement in Sports. Morgantown, WV: M. Weiss.

Galván, J. F.; López-Walle, J.; Pérez, J. A.; Tristán, J. L., y Medina, R. E. (2013). Clima motivacional en deportes individuales y de conjunto en atletas jóvenes mexicanos. Revista Iberoamericana de Psicología del Ejercicio y el Deporte, 8(2), 393-410.

García, E.; Pérez, J.; Rodríguez, P.; Moral, J., y Agudo, F. (2013). La coordinación segmentaria y la agilidad como variables predictoras de práctica deportiva federada en adolescentes. Trances, 5(4), 281-298.

Gavita, E. (2006). Motivos de participación y satisfacción en la actividad física, el ejercicio físico y el deporte. Revista MHSalud, 3(1), 1-16.

Gil, P.; Cuevas, R.; Contreras, O., y Díaz, A. (2012). Educación Física y hábitos de vida activa: percepciones de los adolescentes y relación con el abandono deportivo. Aula Abierta, 40(3), 115-124.

González, J., y Portolés, A. (2014). Actividad física extraescolar: relaciones con la motivación educativa, rendimiento académico y conductas asociadas a la salud. Revista Iberoamericana de Psicología del Ejercicio y el Deporte, 9(1), 51-65.

González, J.; Garcés, E. J., y García, A. (2012). Indicadores de bienestar psicológico percibido en alumnos de Educación Física. Revista de Psicología del Deporte, 21(1), 183-187.

González-Cutre, D.; Sicilia, A., y Moreno, J. A. (2008). Modelo cognitivo-social de la motivación de logro en educación física. Psicothema, 20(4), 642-651.

González-Cutre, D.; Sicilia, A., y Moreno, J. (2011). Un estudio cuasi-experimental de los efectos del clima motivador tarea en las clases de Educación Física. Revista de Educación, 365(1), 677-700. 
Gutiérrez, M.; Ruiz, L. M., y López, E. (2011). Clima motivacional en Educación Física: concordancia entre las percepciones de los alumnos y las de sus profesores. Revista de Psicología del Deporte, 20(2), 321-335.

Hills, A.; King, N., \& Armstrong, T. (2007). The Contribution of Physical Activity and Sedentary Behaviours to the Growth and Development of Children and Adolescents Implications for Overweight and Obesity. Sports Medicine, 37(6), 533-545. http://dx.doi.org/10.2165/00007256-200737060-00006

Isorna, M.; Rial, A., y Vaquero-Cristóbal, R. (2014). Motivaciones para la práctica deportiva en escolares federados y no federados. Retos: nuevas tendencias en educación física, deporte y recreación, 25, 80-84.

Janssen, I.; Katzmarzyk, P.; Boyce, W.; Vereecken, C.; Mulvihill, C.; Roberts, C.; Currie, C., \& Pickett, W. (2005). Comparision of overweight and obesity prevalence in school-aged youth from 34 countries and their relationships with physical activity and dietary patterns. Obesity Reviews, 6, 123-132.

http://dx.doi.org/10.1111/j.1467-789X.2005.00176.x

Kilpatrick, M.; Hebert, E., \& Bartholomew, J. (2015). College Student's Motivation for Pfhysical Activity: Differentiating Men's and Women's Motives for Sport Participation and Exercise. Journal of American College Health, 54(2), 87-94.

http://dx.doi.org/10.3200/JACH.54.2.87-94

López-Alameda, S.; Alonso-Benavente, A.; López-Ruiz de Salazar, A.; Miragaya-López, P.; Alonso-del Olmo, J. A., y González-Herranz, P. (2012). Enfermedad de SindingLarsen-Johansson: análisis de factores asociados. Revista Española de Cirugía Ortopédica y Traumatología, 56(5), 354-360.

http://dx.doi.org/10.1016/j.recot.2012.05.004

Luengo, C. (2007). Actividad físico-deportiva extraescolar en alumnos de primaria. Revista Internacional de Medicina y Ciencias de la Actividad Física y el Deporte, 7(27), 174-184.

Martín-Albo, J. (2000). La motivación en los deportes de equipo: análisis de las motivaciones de inicio, mantenimiento, cambio y abandono. Un programa piloto de intervención. Revista Digital Lecturas: Educación Física y Deportes, 24, 245-248.

Martínez, C.; Cervelló, E., y Moreno, J. A. (2012). Predicción de las razones del alumnado para ser disciplinado en educación física. Revista Latinoamericana de Psicología, 44(3), 41-52.

McDavid, L.; Cox, A., \& Amorose, A. (2011). The relative roles of physical education teacherss and parents in adolescent's leisure-time physical activity motion and behaviour. Psychology of Sport and Exercise, 12(2), 99-107.

Méndez-Giménez, A.; Cecchini, J. A.; Fernández-Río, J., y González, C. (2012). Autodeterminación y metas sociales: un modelo estructural para comprender la intención de práctica, el esfuerzo y el aburrimiento en Educación Física. Aula Abierta, $40(1), 51-62$.

Méndez-Giménez, A.; Fernández-Río, J., y Cecchini-Estrada, J. A. (2014). Análisis de un modelo multiteórico de metas de logro, metas de amistad y autodeterminación en educación física. Estudios de Psicología, 33(29), 325-336.

Méndez-Giménez, A.; Fernández-Rio, J., y Cecchini-Estrada, J. A. (2013). Climas motivacionales, necesidades, motivación y resultados en Educación Física. Aula Abierta, 41(1), 63-72.

Mora, A.; Cruz, J., y Sousa, C. (2015). Cómo mejorar el clima motivacional y los estilos de comunicación en el ámbito de la Educación Física y el deporte. Journal for the Study of Education and Development, 36(1), 91-103. 
Moreno, B.; Jiménez, R.; Gil, A.; Aspano, M., y Torreno, F. (2011). Análisis de la percepción del clima motivacional, necesidades psicológicas básicas, motivación autodeterminada y conductas de disciplina de adolescentes en las clases de educación física. European Journal of Human Movementent, 26(1), 1-24.

Moreno, J. A. (1999). Motricidad Infantil. Aprendizaje y desarrollo a través del juego. Murcia: DM.

Moreno, J. A., y Hellín, M. G. (2007). El interés del alumnado de Educación Secundaria Obligatoria hacia la Educación Física. Revista Electrónica de Investigación Educativa, 9(2), 1-20.

Moreno, J. A.; Cervelló, E. M., \& González-Cutre, D. (2007). Young Athletes' Motivational Profiles. Journal of Sports Science and Medicine, 6, 172-179.

Moreno-Murcia, J. A.; Cervelló-Gimeno, E., \& González-Cutre, D. (2008). Relationships among goal orientations, motivational climate and flow in adolescent athletes: differences by gender. The Spanish Journal of Psychology, 11, 181-191. http://dx.doi.org/10.1017/S1138741600004224

Moreno-Murcia, J. A.; Sicilia, A.; Sáenz-López, P.; González-Cutre, D.; Almagro, B. J., y Conde, C. (2014). Análisis motivacional comparativo en tres contextos de actividad física. Revista Internacional de Medicina y Ciencias de la Actividad Física y el Deporte. 14(56), 665-685.

Moreno-Murcia, J. A.; Zomeño, T.; Marín, L. M.; Ruíz, L. M., y Cervelló, E. (2013). Percepción de la utilidad e importancia de la educación física según la motivación generada por el docente. Revista de Educación, 362(1), 380-401.

Morgan, K.; Kingston, K., \& Sproule, J. (2005). Effects of different teaching styles on the teacher behaviours that influence motivational climate and pupil's motivation in physical education. European Physical Education Review, 11(3), 257-285. http://dx.doi.org/10.1177/1356336X05056651

Newton, M.; Duda, J. L., \& Yin, Z. (2000). Examination of the psychometric properties of the Perceived Motivational Climate in Sport Questionnaire-2 in a sample of female athletes. Journal of Sports Sciences, 18, 275-290.

http://dx.doi.org/10.1080/026404100365018

Oviedo, G.; Sánchez, J.; Castro, R.; Calvo, M.; Sevilla, J. C.; Iglesias, A., y Guerra, M. (2013). Niveles de actividad física en población adolescente: estudio de caso. Retos: nuevas tendencias en educación física, deporte y recreación, 23, 43-47.

Owen, K.; Astell-Burt, T., \& Lonsdale, C. (2013). The relationship between selfdetermined motivation and physical activity in adolescent boys. Journal of Adolescent Health, 53, 420-423.

http://dx.doi.org/10.1016/j.jadohealth.2013.05.007

Pedrosa, I.; García-Cueto, E.; Suárez-Álvarez, J., y Pérez, B. (2012). Adaptación española de una escala de apoyo social percibido para deportistas. Psicothema, 24(3), 470-476.

Reigal, R.; Videra, A.; Parra, J. L., y Juárez, R. (2012). Actividad físico deportiva, autoconcepto físico y bienestar psicológico en la adolescencia. Retos: Nuevas tendencias en Educación Física, Deporte y Recreación, 22(1), 19-23.

Revuelta, L., y Esnaola, I. (2011). Clima familiar deportivo y autoconcepto físico en la adolescencia. European Journal of Education and Psychology, 4(1), 19-31. 
Rodríguez, A.; De la Cruz, E.; Feu, S., y Martínez, R. (2012). Sedentarismo, obesidad y salud mental en la población española de 4 a 15 años de edad. Revista Española de Salud Pública, 85(4), 373-382.

http://dx.doi.org/10.1590/S1135-57272011000400006

Ruiz, F.; García, M. E., y Hernández, A. I. (2001).Comportamientos de actividades físico-deportivas de tiempo libre del alumnado almeriense de enseñanza secundaria postobligatoria. Revista Motricidad, 7, 113- 143.

Salazar, C. M.; Feu, S.; Vizuete, M., y De la Cruz-Sánchez, E. (2013). Entorno social y afectivo y entorno urbano como determinantes del patrón de actividad física de los universitarios de Colima. Cultura, Ciencia y Deporte, 23(8), 103-112.

Sallis, J.; McKenzie, T. L.; Beets, M. W.; Beighle, A.; Erwin, H., \& Lee, S. (2012). Physical education's role in public health: Steps forward and backward over 20 years and hope for the future. Research Quarterly for Exercise and Sport, 83(2), 125-135. http://dx.doi.org/10.1080/02701367.2012.10599842

Solá, I.; Fernández, I.; Ródenas, L.; Fabra, P., y Duda, J. L. (2015). Los entrenadores como promotores de la cohesión del equipo. Cuadernos de psicología del deporte, 15(1), 233-242.

http://dx.doi.org/10.4321/S1578-84232015000100022

Spittle, M., \& Byrne, K. (2009). The influence of sport education on student motivation in physical education. Physical Education and Sport Pedagogy, 14(3), 253-266. http://dx.doi.org/10.1080/17408980801995239

Standage, M.; Gillison, F. B.; Ntoumanis, N., \& Treasure, D. (2012). Predicting student's physical activity and health-related well-being: A prospective cross-domain investigation of motivation across school physical education and exercise settings. Journal of Sport \& Exercise Psychology, 34, 37-60.

Strong, W.; Malina, R.; Blimkie, C.; Daniels, S.; Dishman, R.; Gution, B.; Hergenroeder, A. C.; Must, A.; Nixon, P. A.; Pivarnik, J. M.; Rowland, T.; Trost, S., \& Trudeau, F. (2005). Evidence based physical activity for school-age youth. Journal of Pediatrics, $146,732-737$.

http://dx.doi.org/10.1016/j.jpeds.2005.01.055

Teixeira, P.; Carraça, E.; Markland, D.; Silva, M., \& Ryan, R. (2012). Exercise, physical activity, and self-determination theory: A systematic review. International Journal of Behavioural Nutrition and Physical Activity, 9(1), 78-108.

http://dx.doi.org/10.1186/1479-5868-9-78

Torregrosa, M.; Sousa, C.; Viladrich, C.; Villamarín, F., y Cruz, J. (2008). El clima motivacional y el estilo de comunicación del entrenador como predictores del compromiso en futbolistas jóvenes. Psicothema, 20, 254-259.

Torregrosa, M.; Viladrich, C.; Ramis, Y.; Azócar, F.; Latinjak, A., y Cruz, J. (2011). Efectos en la percepción del clima motivacional generado por los entrenadores y compañeros sobre la diversión y el compromiso. Diferencias en función del género. Revista de Psicología del Deporte, 20(1), 243-255.

Van Laar, C.; Derks, B., \& Ellemers, N. (2013). Motivation for education and work in Young Muslim women: The importance of value for ingroup domains. Basic and Applied Social Psychology 35(1), 64-74. http://dx.doi.org/10.1080/01973533.2012.746609

Vazou, S.; Ntoumanis, N., \& Duda, J. L. (2006). Predicting young athletes' motivational indices as afunction of their perceptions of the coach- and peer-created climate. Psychology of Sport and Exercise, 7, 215-233. http://dx.doi.org/10.1016/j.psychsport.2005.08.007 
Castro-Sánchez, M.; Zurita-Ortega, F.; Martínez-Martínez, A.; Chacón-Cuberos, R., y Espejo-Garcés, T. (2016). Clima motivacional de los adolescentes y su relación con el género, la práctica de actividad física, la modalidad deportiva, la práctica deportiva federada y la actividad física familiar. RICYDE. Revista internacional de ciencias del deporte. 45(12), 262-277. http://dx.doi.org/10.5232/ricyde2016.04504

Wallhead, T. L.; Garn, A. C., \& Vidoni, C. (2014). Effect of a Sport Education Program on Motivation for Physical Education and Leisure-Time Physical Activity. Research quarterly for exercise and sport, 85(4), 478-487). http://dx.doi.org/10.1080/02701367.2014.961051

Warburton, D.; Whitney, C., \& Bredin, S. (2006). Health benefits of physical activity: the evidence. Canadian Medical Association Journal, 174(6), 801-809. http://dx.doi.org/10.1503/cmaj.051351

Zurita, F.; Fernández, R.; Cepero, M.; Zagalaz, M. L.; Valverde, M., \& Ramírez, P. (2009). The relationship between pain and physical activity in older adults that begin a program of physical activity. Journal of Human Sport and Exercise, 4(3), 248-297. http://dx.doi.org/10.4100/jhse.2009.43.10 